\title{
Effects of fecal microbiota transplantation on serum uric acid levels, symptoms and intestinal barrier function in patients with acute and recurrent gout: a pilot study
}

\section{Wenrui Xie}

First Affiliated Hospital of Guangdong Pharmaceutical Universtiy

\section{Xiaoya Yang}

Guangzhou Health Science College

\section{Zhihe Deng}

First Affiliated Hospital of Guangdong Pharmaceutical University

\section{Yamei Zheng}

First Affiliated Hospital of Guangdong Pharmaceutical University

\section{Ran Zhang}

First Affiliated Hospital of Guangdong Pharmaceutical University

Lihao Wu

First Affiliated Hospital of Guangdong Pharmaceutical University

Jieyi Cai

First Affiliated Hospital of Guangdong Pharmaceutical University

\section{Harry Huaxiang Xia}

First Affiliated Hospital of Guangdong Pharmaceutical University

Xingxiang He ( $\nabla$ hexingxiang@gdpu.edu.cn )

First Affiliated Hospital of Guangdong Pharmaceutical University https://orcid.org/0000-0003-00078513

\section{Research article}

Keywords: gout, uric acid, fecal microbiota transplantation, gut microbiota, intestinal barrier

Posted Date: July 15th, 2020

DOI: https://doi.org/10.21203/rs.3.rs-41655/v1

License: (c) (i) This work is licensed under a Creative Commons Attribution 4.0 International License. 

1 Effects of fecal microbiota transplantation on serum uric acid levels,

2 symptoms and intestinal barrier function in patients with acute and

3 recurrent gout: a pilot study

4

5 Wenrui Xie ${ }^{1, \#}$, Xiaoya Yang ${ }^{2, \#}$, Zhihe Deng ${ }^{1}$, Yamei Zheng ${ }^{1}$, Ran Zhang ${ }^{1}$, Lihao $\mathrm{Wu}^{1}$,

6 Jieyi Cai ${ }^{1}$, Harry Huaxiang $\mathrm{Xia}^{1,{ }^{1}}$, Xingxiang $\mathrm{He}^{1,{ }^{*}}$

7

81 Department of Gastroenterology, The First Affiliated Hospital of Guangdong

9 Pharmaceutical University, Guangzhou 510080, China

2 Department of Physiology, Guangzhou Health Science College, Guangzhou 510180,

11 Guangdong Province, China

12

13

14

15

\#Wenrui Xie and Xiaoya Yang contributed equally to this work

\section{* Correspondence:}

Harry Huaxiang Xia and Xingxiang He

Address: 19 Nonglinxia Road, Guangzhou 510080, Guangdong Province, China.

E-mail: xiaharry@hotmail.com (Harry Huaxiang Xia), hexingxiang@gdpu.edu.cn

(Xingxiang $\mathrm{He}$ ) 


\section{Abstract}

2 Background: Gut dysbiosis has been reported to be closely associated with gout. Fecal

3 microbiota transplantation (FMT) has been considered as an effective way to restore

4 the balance of gut microbiota. We aimed to evaluate the effects of FMT on serum uric

5 acid levels, symptoms and the intestinal barrier function in patients with acute and

6 recurrent gout.

7 Methods: We performed a pilot study of FMT for acute and recurrent gout. The primary

8 outcome was the changes in serum uric acid level on day 28 post-FMT and in gout

9 symptoms by one year. The secondary outcomes included the changes in levels of urine uric acid, diamine oxidase (DAO), D-lactic acid and endotoxin on day 28 post-FMT.

11 The levels of DAO, D-lactic acid and endotoxin were assessed by enzyme assay.

Results: Eleven patients received FMT treatment. All the patients had a reduction in serum uric acid levels after FMT treatment $(P<0.05)$, accompanied with a decrease in the frequency and duration time of acute gout flares. The levels of DAO, D-lactic acid and endotoxin, reflecting the intestinal barrier function, were higher in patients with gout than in healthy donors $(P<0.05)$. After FMT treatment, the levels of DAO and endotoxin decreased $(P<0.05)$.

Conclusions: FMT is effective for reducing serum uric acid levels and improving gout symptoms in patients with gout, and contributes to improve the impaired intestinal barrier function of the patients.

Trial registration: Chinese Clinical Trial Registry, ChiCTR2000034584. Registered 
1 http://www.chictr.org.cn/showproj.aspx?proj=55871

2 Keywords: gout; uric acid; fecal microbiota transplantation; gut microbiota; intestinal

3 barrier

4

5

6

7

8

9

10

11

12

13

14

15

16

17

18

19

20

21

22 
2 Gout, the most common form of inflammatory arthritis, is a chronic urate crystal

3 deposition disease [1]. Sustained hyperuricemia is the uppermost risk factor for gout,

4 mainly caused by the overproduction and/or underexcretion of urate [1].

5 Underexcretion of urate is the primary cause of hyperuricemia for most patients with

6 gout. The urate is mainly excreted through the kidney and intestine pathways,

7 accounting for two thirds and one third of the total urate, respectively. In case of renal

8 damage, the intestine pathway will compensatorily become the main pathway to

9 eliminate urate [1].

10 Gut microbiota, crucial for maintaining the homeostasis of the intestinal 11 environment, is involved in the metabolism of the host [2]. It has been reported that the 12 gut dysbiosis exists in patients with gout [3,4], which leads to the abnormal metabolism 13 of host purine and uric acid [5]. Manipulation of gut dysbiosis with probiotics alleviates 14 fructose-induced hyperuricemia in mice, accompanied with the enhancement of 15 intestinal barrier function [6]. Therefore, a possibility arises that the patients with gout 16 will benefit from restoring a healthy gut microbiota.

Fecal microbiota transplantation (FMT) has been regarded as a safe and effective 18 way to restore a healthy gut microbiota, exhibiting remarkable clinical efficacy for 19 Clostridium difficile infection, inflammatory bowel disease and other gut dysbiosis20 related diseases [7-10]. Recently, Han et al. conducted FMT in hyperuricemic mice, 21 and found that serum uric acid levels reduced and uric acid excretion increased [11], 22 demonstrating an ameliorating effect of FMT on hyperuricemia. They further found that 
1 the expression of claudin-1 and occludin in these mice was increased, compared to that

2 in hyperuricemic mice with renal injury [11]. These data indicate that FMT repairs

3 intestinal integrity, which might function as one of the underlying mechanisms by

4 which FMT alleviates hyperuricemia. Although the encouraging results from the animal

5 experiments, whether FMT reduces the serum uric acid levels and improves gout

6 symptoms in patients with gout and improves intestinal integrity in human remains

7 unknown.

8 In this pilot study, we aimed to evaluate the effects of FMT on serum uric acid

9 levels, symptoms and the intestinal barrier function in patients with acute and recurrent 10 gout.

\section{Methods}

\section{Subjects}

14 This study was conducted in the First Affiliated Hospital of Guangdong Pharmaceutical 15 University from June 2017 to June 2019. Written informed consent was obtained from 16 all subjects or guardians. The study protocol was reviewed and approved by the Ethical 17 Committee of the First Affiliated Hospital of Guangdong Pharmaceutical University 18 (No. 201798).

\section{Inclusion and exclusion criteria}

20 Patients who met the following criteria were included in the study: 1) aged $\geq 18$ years;

21 2) diagnosed with gout according to the Clinical Gout Diagnosis Criteria [12]; and 3) 
1 treated with uric-acid-lowering drugs for more than one year, but still with

2 hyperuricemia and suffering from acute gout flares twice or more a year. The exclusion

3 criteria were as follows: 1) pregnancy; 2) gastrointestinal infection, cardiopulmonary

4 failure or serious liver diseases; and 3) rejection to conduct transendoscopic enteral

5 tubing. There was no sex restriction.

6 The healthy donors without kinship to patients with gout were included as the

7 comparison group. All the donors were strictly screened by the selection criteria

8 formulated by Zhang's group [13, 14]. The exclusion criteria for the healthy donors

9 were as follows: 1) a history of drug use during the last 3 months; 2) a history of

10 infectious diseases (during the last 3 months), hereditary diseases, metabolic diseases,

11 or any other diseases associated with alterations in gut microbiota; and 3) laboratory

12 abnormalities.

\section{FMT procedure}

14 The fresh fecal microbiota suspension was prepared using the automatic purification 15 system (GenFMTer, China). The prepared fresh fecal microbiota suspension was 16 infused into the intestines of patients within $1 \mathrm{~h}$ after isolation, based on the "one-hour 17 FMT protocol" [15]. Transendoscopic enteral tubing was employed to deliver the suspension through the lower digestive tract by colonoscope or through the upper 19 digestive tract by gastroscope. Suspension $(200 \mathrm{~mL})$ was infused each time, every other 20 day for 3 consecutive times as a course of treatment. During FMT, no adjustment was 21 made to the drugs that patients had been taking for gout. 
1 and 28 for the occurrence of adverse events (AEs), and days 90, 180 and 365 for the

2 frequency and duration time of acute gout flares.

34 Assays for uric acid, diamine oxidase (DAO), D-lactic acid and endotoxin

4 For patients, assays for uric acid, DAO, D-lactic acid and endotoxin were performed on

5 the day before FMT and on day 28 post-FMT. For donors, assays were performed on

6 the day they donated fecal microbiota. The levels of serum and urine uric acid were

7 determined using uric acid kit (Biosino Bio-technology and Science Inc, China) with

8 an automatic biochemical analyzer (Hitachi, Japan), following the manufacture's

9 protocols. The levels of DAO, D-lactic acid and endotoxin were assessed by enzyme

10 assay using the diamine oxidase, lactic acid, bacterial endotoxin assay kit

11 (ZhongShengJinYu, China), according to the manufacture's protocols. The tests were

12 conducted on the analysis system of biochemical indicators of intestinal barrier function

13 (JY-DLT, ZhongShengJinYu, China).

\section{Clinical outcomes}

15 The primary outcome was the changes in serum uric acid levels on day 28 post-FMT and the frequency and duration time of acute gout flares by one year. The secondary

17 outcomes included the changes in levels of urine uric acid, DAO, D-lactic acid and endotoxin on day 28 post-FMT, and occurrent of AEs within 28 post-FMT. We graded

19 the AEs according to the NCI Common Terminology Criteria for Adverse Events 20 version 5.0, and classified the relation between AEs and FMT as unrelated, possibly, 21 probably and definitely related [16]. 
1 Normally distributed data were presented as mean \pm standard deviation (SD), while

2 non-normally distributed data were presented as median (interquartile range).

3 Comparisons of the differences before and after FMT treatment were performed using

4 paired-samples $t$-test for the normally distributed data, or Wilcoxon Signed Rank Test,

5 otherwise. Comparisons of the differences between the patients and donors were

6 performed using independent-samples $t$-test for the normally distributed data with equal

7 variance, or Mann-Whitney U-test, otherwise. SPSS statistical package (version 19.0)

8 was employed to perform all statistical analyses. $P<0.05$ was regarded as statistical

9 significance.

\section{Results}

\section{The baseline data of patients with gout and donors}

13 Eleven patients with gout and six donors were included. The baseline characteristics of 14 the patients and donors are exhibited in Table 1. All patients suffered from recurrent acute gout flares (2 - 5 times per year), and one patient had gouty tophi. In terms of comorbidities, all patients suffered from hyperuricemia; seven had nonalcoholic fatty

17 liver disease, but none had kidney disease.

\section{Reduction of serum uric acid levels in patients with gout after FMT}

19 Serum uric acid levels in patients with gout before FMT was $(592.54 \pm 126.82) \mu \mathrm{mol} / \mathrm{L}$,

20 which were significantly higher than that in donors (Mann-Whitney $U$ value $=0.000$, $21 P=0.001 ;$ Fig. 1). 
After FMT, the serum uric acid levels reduced in eight (72.73\%) out of 11 patients.

2 The averaged serum uric acid levels in patients reduced to $(481.55 \pm 119.77) \mu \mathrm{mol} / \mathrm{L}$,

3 which was significantly lower than that before FMT $(t=2.513, P=0.031$; Fig. 1$)$, but

4 still significantly higher than that in donors (Mann-Whitney $\mathrm{U}$ value $=11.000, P=$ $5 \quad 0.031$; Fig. 1).

$6 \quad$ Urine uric acid test results before and after FMT were available in four of the 11

7 patients. As shown in Table S1, the urine uric acid levels decreased in three patients but

8 increased in one patient after FMT; there was no significant difference between the 9 levels before and after FMT $(2164.75 \pm 1651.51$ vs. $1773.00 \pm 558.41, t=0.706, P=$ $10 \quad 0.531)$.

113 Improvement of gout symptoms and occurrence of AEs after FMT

All patients received FMT treatment for one to three courses: six for one course, four for two courses and one for three courses. The microbiota suspension was delivered through the lower digestive tract by colonoscope in eight patients, and through the upper digestive tract by gastroscope in three patients. All patients reported a decrease in the frequency and duration time of acute gout flares after FMT treatment. Two patients who experienced acute gout flares 2 - 3 times per year before FMT, reported no gout flares during the one-year follow-up after two or three courses of FMT.

Two mild AEs, anal discomfort and abdominal distention, occurred during FMT and were resolved within one week. These AEs were classified as possibly related to FMT. No AEs occurred during the 28-day period of follow-up. 


\section{after FMT}

As shown in Fig. 2, the levels of serum DAO, D-lactic acid and endotoxin in patients with gout were higher than those in donors, indicating the decline in intestinal barrier function in patients with gout.

After FMT treatment, the levels of serum DAO and endotoxin in patients with gout were reduced $(P<0.05$; Fig. 2A and C). D-lactic acid level decreased slightly, with no significant difference ( $t=1.237, P=0.244$; Fig. 2B). In addition, there were no significant differences in the levels of serum DAO and endotoxin between patients with gout after FMT and the donors (Fig. 2A and C), indicating that the levels of serum DAO and endotoxin return to normal after FMT treatment. However, D-lactic acid levels in patients with gout after FMT was still obviously higher than that in donors (MannWhitney $\mathrm{U}$ value $=3.000, P=0.003$; Fig. 2B).

\section{Discussion}

In this pilot study, we found that the serum uric acid levels in patients with acute and recurrent gout were reduced and their gout symptoms were improved after FMT treatment. We further found that their impaired intestinal barrier function was improved after FMT.

Previous studies have demonstrated that gut microbiota plays a crucial role in degradation of uric acid [17], and gut dysbiosis is present in patients with gout $[3,4]$, which may lead to the decline in degradation of uric acid and the subsequent elevation 
1 of serum uric acid level. Consistent with these studies, we found that serum uric acid

2 was increased in all patients with acute and recurrent gout in our study.

FMT has been considered as a safe and efficient way to manipulate gut dysbiosis [7-10]. In this study, we demonstrated that FMT reduced serum uric acid levels in patients with gout, which is similar to the observations of the effects of FMT in mice with hyperuricemia [11]. Moreover, all patients in our study reported decreased frequency and duration time of acute gout flares after FMT, suggesting that the clinical symptoms were improved and FMT might function as a new treatment option for gout patients.

The primary cause of hyperuricemia for most people with gout is underexcretion of urate [1]. The kidney and intestine are the main excretion pathways of uric acid $[2,18]$. In the present study, the urine uric acid levels in gout patients seemed to be unchanged after FMT treatment, suggesting that FMT might not affect the excretion of uric acid through the kidney, and the reduced serum acid levels after FMT might not be caused by the increased elimination through the kidney, but associated with an increased elimination through the intestine. However, the data on the urine uric acid levels before and after FMT were available only in four patients, and thus our observation needs to be confirmed in studies with more sample sizes.

In the experiments in mice with hyperuricemia, it has been found that hyperuricemia damages the intestinal barrier function, leading to the increased intestinal permeability $[19,20]$. In our present study, the levels of DAO, D-lactic acid and endotoxin in patients with gout increased, indicating the intestinal barrier function was impaired. Noticeably, 
1 the levels of DAO and endotoxin in patients with gout reduced to a similar level with

2 the donors after FMT administration in our study, suggesting that FMT treatment may

3 restore the intestinal barrier function to the normal level. Based on the findings in the

4 animal studies and our observation in the present study, we would propose that FMT

5 administration might reduce serum uric acid levels in patients with gout via increasing

6 the elimination uric acid by the rebalanced gut microbiota; the reduced uric acid level

7 might contribute to the improvement of intestinal barrier function.

The D-lactic acid level was elevated in patients with gout, which was only slightly decreased after FMT. Recently, Drabkin et al. found that a missense mutation in lactate dehydrogenase D (LDHD) led to the elevated levels of D-lactate, which resulted in hyperuricemia and gout ultimately [21]. A plausible explanation for the unchanged Dlactic acid levels in the patients with gout after FMT observed in our study might be that the missense mutation in $L D H D$ existed in the patients, leading to the elevated levels of D-lactic acid. FMT administration restored the balance of gut microbiota, but could not repair the missense mutation in $L D H D$. Thus, D-lactic acid remained at a high level even after FMT. Further investigation is required to confirm this assumption. inflammasome is activated by interaction between monosodium urate crystals and macrophages, which leads to the maturation of IL-1 $\beta$ through recruiting caspase 1 [22]. 
1 Lipopolysaccharides (LPS), also known as lipoglycans and endotoxins, have been

2 reported to activate NLRP3 inflammasome and release the mature IL-1 $\beta$ through

3 releasing endogenous ATP from monocytes, and the following activation of P2X7

4 receptor [23]. LPS can also increase the expressions of NLRP3 protein and pro-IL-1 $\beta$

5 in macrophages [24]. Therefore, LPS may play a promoting role in the acute gout flares.

6 In our study, we demonstrated that the endotoxin level in patients with gout was

7 elevated. After FMT treatment, the endotoxin level returned to normal, accompanied

8 with the reduced frequency and duration time of acute gout flares. These findings

9 indicate that FMT contributes to the reduced acute gout flares, likely through pathways 10 involving endotoxins.

11 It has been reported that gut dysbiosis and the intestinal barrier impairment are both 12 involved in the pathogenesis of many diseases [25-28]. However, it is unclear which of 13 them acts as "cause" and which acts as "effect". Whereas some studies report that gut 14 dysbiosis affects the intestinal mucosal barrier [26-28], others demonstrate that the 15 impaired intestinal barrier function as an initiating factor for gut dysbiosis [29-31]. 16 Therefore, an interplay between gut dysbiosis and the intestinal barrier impairment may 17 contribute to the development of diseases. Gut dysbiosis has been found existing in 18 patients with gout $[3,4]$. In this study, we demonstrated that the intestinal barrier 19 function was impaired in patients with gout, which was improved after FMT. These 20 observations indicate that an interplay or positive feedback between gut dysbiosis and 21 the intestinal barrier impairment may contribute to the development of gout, and restoration of a healthy gut by FMT breaks the interplay or positive feedback, reduces 

21 of the patients.

\section{Conclusions}

serum uric acid and improves the intestinal barrier function.

A couple of limitations existed in this study. First, as mentioned earlier, the cohort size is small, with only 11 in gout group and six in donor group. The test results of the urine uric acid levels before and after FMT were available only for four patients, and thus the sample size was too small to reach the statistical significance and draw a conclusion. Second, all subjects included in this study were male, possibly because of the high prevalence of gout in men. It remains unknown whether FMT will show the similar efficacy in female patients. Third, there was no positive control group (i.e. patients with gout but without FMT) in the study. In the present study, no adjustment was made for drugs treating gout was made. It is unknown whether the alleviation in gout symptoms and the reduction in serum uric acid levels observed were solely resulted from FMT treatment, or a combination of the drugs and FMT. Therefore, large and well-designed clinical trials are necessary to verify the efficacy of FMT in patients with gout. In addition, further investigation on the alterations of the composition of gut microbiota in patients with gout before and after FMT, is required to determine the relationship among gut dysbiosis, serum uric acid and intestinal barrier function.

FMT is effective for reducing serum uric acid levels and improving gout symptoms in patients with gout. FMT contributes to improve the impaired intestinal barrier function 
2 Abbreviations

3 FMT: Fecal microbiota transplantation; DAO: Diamine oxidase; AEs: adverse events;

$4 \quad$ LDHD: lactate dehydrogenase D; LPS: Lipopolysaccharides

6 References

7 1. Dalbeth N, Merriman TR, Stamp LK. Gout. Lancet. 2016;388(10055):2039-52.

8 2. Pan L, Han P, Ma S, Peng R, Wang C, Kong W, et al. Abnormal metabolism of gut

9 microbiota reveals the possible molecular mechanism of nephropathy induced by

10 hyperuricemia. Acta pharmaceutica Sinica B. 2020;10(2):249-61.

11 3. Guo Z, Zhang J, Wang Z, Ang KY, Huang S, Hou Q, et al. Intestinal microbiota 12 distinguish gout patients from healthy humans. Scientific reports. 2016;6:20602.

4. Shao T, Shao L, Li H, Xie Z, He Z, Wen C. Combined signature of the fecal 14 microbiome and metabolome in patients with gout. Frontiers in microbiology. $15 \quad 2017 ; 8: 268$.

5. Chiaro TR, Soto R, Zac Stephens W, Kubinak JL, Petersen C, Gogokhia L, et al. A 17 member of the gut mycobiota modulates host purine metabolism exacerbating colitis in 18 mice. Science translational medicine. 2017;9(380):eaaf9044.

6. Wang H, Mei L, Deng Y, Liu Y, Wei X, Liu M, et al. Lactobacillus brevis DM9218

20 ameliorates fructose-induced hyperuricemia through inosine degradation and

21 manipulation of intestinal dysbiosis. Nutrition. 2019;62:63-73. 
1 7. Cammarota G, Ianiro G, Tilg H, Rajilic-Stojanovic M, Kump P, Satokari R, et al.

2 European consensus conference on faecal microbiota transplantation in clinical practice.

3 Gut. 2017;66(4):569-80.

4 8. Millan B, Laffin M, Madsen K. Fecal microbiota transplantation: beyond

5 Clostridium difficile. Curr Infect Dis Rep. 2017;19(9):31.

6 9. Bajaj JS, Kassam Z, Fagan A, Gavis EA, Liu E, Cox IJ, et al. Fecal microbiota

7 transplant from a rational stool donor improves hepatic encephalopathy: a randomized

$8 \quad$ clinical trial. Hepatology. 2017;66(6):1727-38.

9 10. Halkjaer SI, Christensen AH, Lo BZS, Browne PD, Gunther S, Hansen LH, et al.

10 Faecal microbiota transplantation alters gut microbiota in patients with irritable bowel

11 syndrome: results from a randomised, double-blind placebo-controlled study. Gut. $12 \quad 2018 ; 67(12): 2107-15$.

13 11. Han J, Wang X, Tang S, Lu C, Wan H, Zhou J, et al. Protective effects of tuna meat 14 oligopeptides (TMOP) supplementation on hyperuricemia and associated renal 15 inflammation mediated by gut microbiota. FASEB journal : official publication of the Federation of American Societies for Experimental Biology. 2020;34(4):5061-76. 12. Vázquez-Mellado J, Hernández-Cuevas CB, Alvarez-Hernández E, Ventura-Rios L, Peláez-Ballestas I, Casasola-Vargas J, et al. The diagnostic value of the proposal for 19 clinical gout diagnosis (CGD). Clinical rheumatology. 2012;31(3):429-34. transplantation strategy: a pilot study for steroid-dependent ulcerative colitis. Journal of translational medicine. 2015;13:298. 
1 14. Cui B, Feng Q, Wang H, Wang M, Peng Z, Li P, et al. Fecal microbiota

2 transplantation through mid-gut for refractory Crohn's disease: safety, feasibility, and

3 efficacy trial results. Journal of gastroenterology and hepatology. 2015;30(1):51-8.

4 15. Zhang F, Zhang T, Zhu H, Borody TJ. Evolution of fecal microbiota transplantation

5 in methodology and ethical issues. Current opinion in pharmacology. 2019;49:11-6.

6 16. Kelly CR, Kunde SS, Khoruts A. Guidance on preparing an investigational new

7 drug application for fecal microbiota transplantation studies. Clinical gastroenterology

8 and hepatology : the official clinical practice journal of the American

9 Gastroenterological Association. 2014;12(2):283-8.

17. Sorensen LB. Role of the intestinal tract in the elimination of uric acid. Arthritis

11 and rheumatism. 1965;8(5):694-706.

18. Singh JA, Ramachandaran R, Yu S, Yang S, Xie F, Yun H, et al. Is gout a risk equivalent to diabetes for stroke and myocardial infarction? A retrospective claims database study. Arthritis research \& therapy. 2017;19(1):228.

19. Guo Y, Li H, Liu Z, Li C, Chen Y, Jiang C, et al. Impaired intestinal barrier function in a mouse model of hyperuricemia. Mol Med Rep. 2019;20(4):3292-300.

20. Xu D, Lv Q, Wang X, Cui X, Zhao P, Yang X, et al. Hyperuricemia is associated with impaired intestinal permeability in mice. American journal of physiology Gastrointestinal and liver physiology. 2019;317(4):G484-g92. Hyperuricemia and gout caused by missense mutation in d-lactate dehydrogenase. The Journal of clinical investigation. 2019;129(12):5163-8. 
22. Martinon F, Petrilli V, Mayor A, Tardivel A, Tschopp J. Gout-associated uric acid crystals activate the NALP3 inflammasome. Nature. 2006;440(7081):237-41.

23. He Y, Hara H, Nunez G. Mechanism and regulation of NLRP3 inflammasome activation. Trends in biochemical sciences. 2016;41(12):1012-21.

24. Liu HJ, Pan XX, Liu BQ, Gui X, Hu L, Jiang CY, et al. Grape seed-derived procyanidins alleviate gout pain via NLRP3 inflammasome suppression. Journal of neuroinflammation. 2017;14(1):74.

25. Ni J, Wu GD, Albenberg L, Tomov VT. Gut microbiota and IBD: causation or correlation? Nature reviews Gastroenterology \& hepatology. 2017;14(10):573-84.

26. Takiishi T, Fenero CIM, Camara NOS. Intestinal barrier and gut microbiota: Shaping our immune responses throughout life. Tissue barriers. 2017;5(4):e1373208.

27. Xue M, Ji X, Liang H, Liu Y, Wang B, Sun L, et al. The effect of fucoidan on intestinal flora and intestinal barrier function in rats with breast cancer. Food \& function. 2018;9(2):1214-23.

28. Ling Z, Liu X, Guo S, Cheng Y, Shao L, Guan D, et al. Role of probiotics in mycoplasma pneumoniae pneumonia in children: a short-term pilot project. Frontiers in microbiology. 2018;9:3261.

29. Yu LC. Microbiota dysbiosis and barrier dysfunction in inflammatory bowel disease and colorectal cancers: exploring a common ground hypothesis. Journal of biomedical science. $2018 ; 25(1): 79$.

30. Saggioro A. Leaky gut, microbiota, and cancer: an incoming hypothesis. Journal of clinical gastroenterology. 2014;48 Suppl 1:S62-6. 
1 31. Lynch SV, Pedersen O. The human intestinal microbiome in health and disease.

2 The New England journal of medicine. 2016;375(24):2369-79.

4 Declarations

5 Ethics approval and consent to participate

6 This study was approved by the Ethical Committee of the First Affiliated Hospital of

7 Guangdong Pharmaceutical University (No. 201798). Written informed consent was

8 obtained from all patients/guardians and donors.

9

\section{Consent for publication}

Not applicable

\section{Availability of data and materials}

The data that support the findings of this study are available on reasonable request from the corresponding author.

\section{Competing interests}

The authors declare that they have no competing interests.

\section{Funding}

This work was supported by Natural Science Foundation of Guangdong Province, 
1 China (2019A1515010125, XXH) and Department of Education of Guangdong

2 Province (2020KZDZX1132, XXH; 2018GKTSCX033, XYY).

3

4 Authors' contributions

5 WRX and XYY designed the study, analyzed the data and wrote the manuscript. ZHD

6 conducted the follow-up and collected the data. YMZ and RZ performed the assays for

7 diamine oxidase, D-lactic acid and endotoxin. LHW and JYC performed the fecal

8 microbiota transplantation and collected the data. HHXX provided professional advice

9 and revised the manuscript. XXH conceived and supervised the study.

\section{Acknowledgements}

We thank the members of Clinical Lab, the First Affiliated Hospital of Guangdong Pharmaceutical University for uric acid assay.

(4)

\section{要}

18
19 


\begin{tabular}{lcc}
\hline & Patients with gout $(\boldsymbol{n}=\mathbf{1 1})$ & Donors $(\boldsymbol{n}=\mathbf{6})$ \\
\hline Gender (Male/female) & $11 / 0$ & $6 / 0$ \\
Age, year & $41.00 \pm 17.64$ & $21.83 \pm 2.14$ \\
Course of disease, year & $4(2-10)$ & 0 \\
\hline Clinical symptoms $(n)$ & 11 & 0 \\
\hline Recurrent acute gouty arthritis & 1 & 0 \\
Gouty tophi & 11 & 0 \\
\hline Comorbidities $(n)$ & 7 & 0 \\
\hline Hyperuricemia & 4 & 0 \\
Nonalcoholic fatty liver disease & & 0 \\
Type 2 diabetes & & 0 \\
\hline
\end{tabular}


$1 \quad$ Figure legends

2 Fig. 1 Reduction of serum uric acid levels in patients with gout after fecal

3 microbiota transplantation (FMT).

4 Serum uric acid levels of donors $(n=6)$ and patients with gout $(n=11)$ before and after

$5 \quad$ FMT. Data are presented as mean \pm standard deviation (SD).

$6 \quad *, P<0.05$, and $* *, P<0.01$, comparison as shown in the figure.

7

8 Fig. 2 Improvement of the impaired intestinal barrier function in patients with

9 gout after fecal microbiota transplantation (FMT).

10 Serum levels of diamine oxidase (A), D-lactic acid (B) and endotoxin (C) in donors ( $n$

$11=6)$ and in patients with gout $(n=11)$ before and after FMT. Data are presented as

12 median (interquartile range) in (A) and (C), as mean \pm standard deviation (SD) in (B).

$13 *, P<0.05$, and $* *, P<0.01$, comparison as shown in the figures. 
Figures

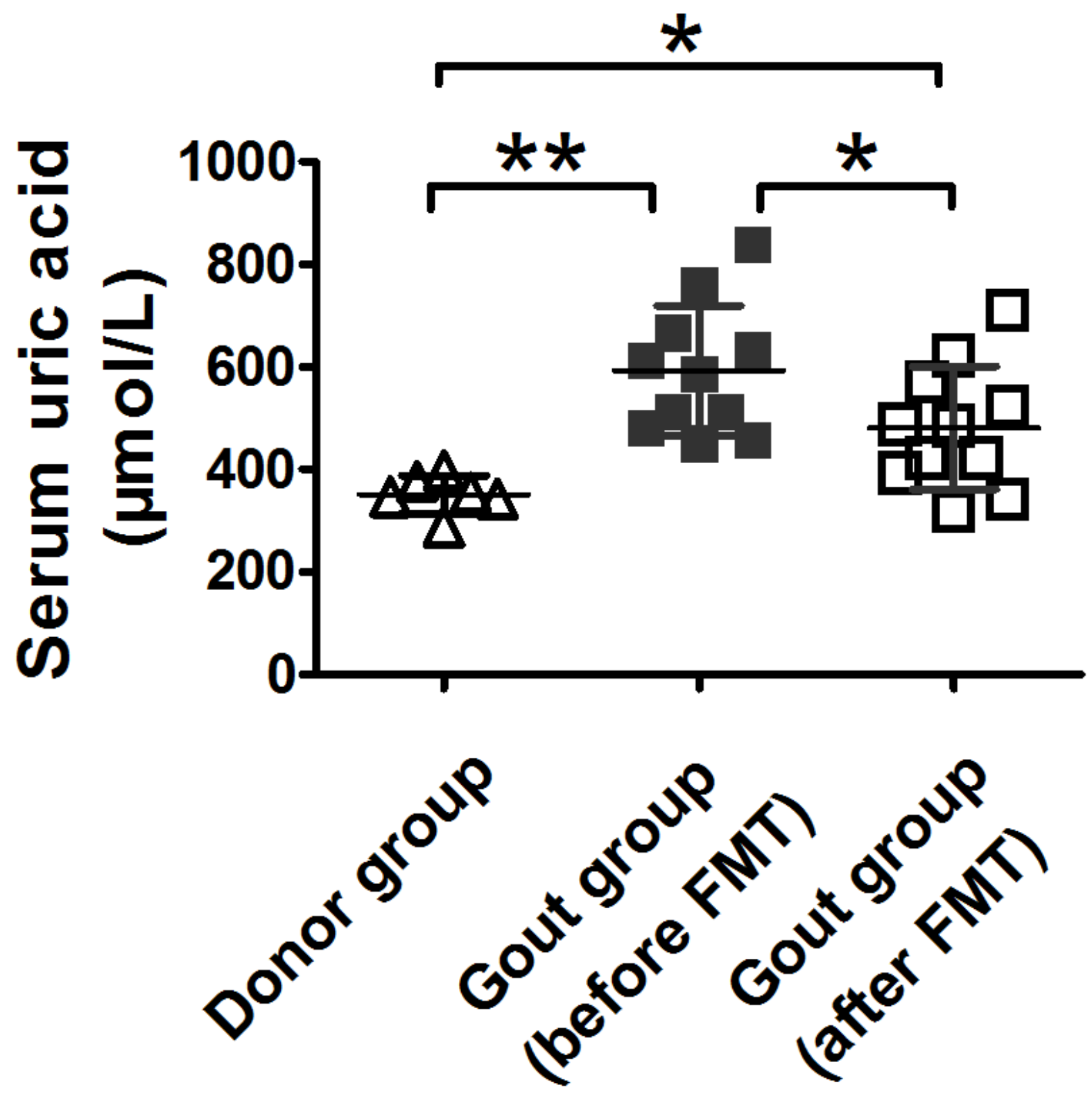

Figure 1

Reduction of serum uric acid levels in patients with gout after fecal microbiota transplantation (FMT). Serum uric acid levels of donors $(n=6)$ and patients with gout $(n=11)$ before and after FMT. Data are presented as mean \pm standard deviation (SD). ${ }^{*}, \mathrm{P}<0.05$, and $* *, \mathrm{P}<0.01$, comparison as shown in the figure. 
A

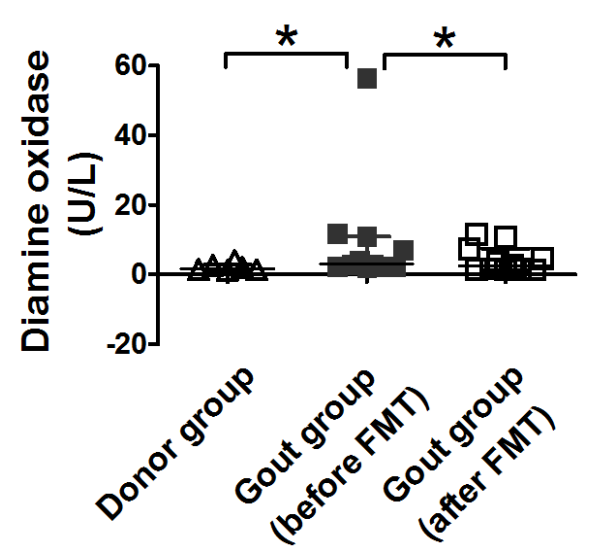

B

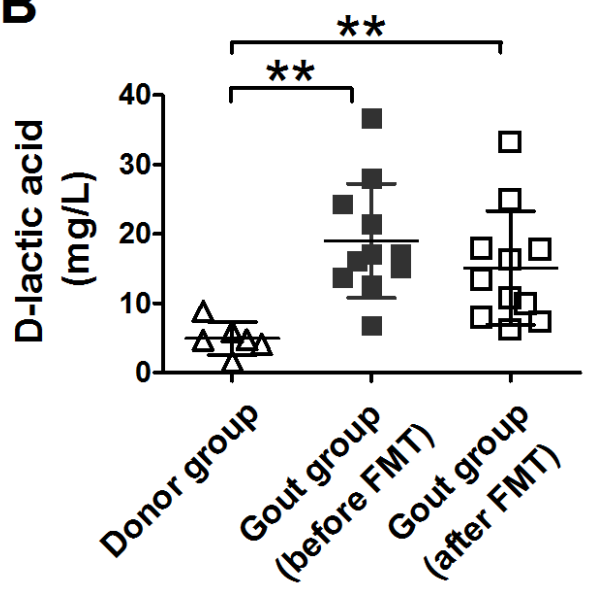

C

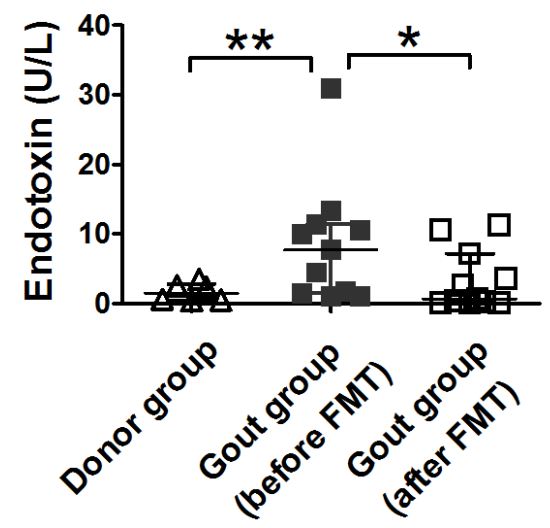

Figure 2

Improvement of the impaired intestinal barrier function in patients with gout after fecal microbiota transplantation (FMT). Serum levels of diamine oxidase (A), D-lactic acid (B) and endotoxin (C) in donors $(n=6)$ and in patients with gout $(n=11)$ before and after FMT. Data are presented as median (interquartile range) in (A) and (C), as mean \pm standard deviation (SD) in (B). * $P<0.05$, and **, $P<0.01$, comparison as shown in the figures.

\section{Supplementary Files}

This is a list of supplementary files associated with this preprint. Click to download.

- TableS1.pdf 